\title{
Paired-associate learning, animacy, and imageability effects in the survival advantage
}

\author{
Stephanie A. Kazanas ${ }^{1}$ • Jeanette Altarriba ${ }^{2}$ • Emily G. O'Brien ${ }^{2}$ \\ Published online: 8 January 2020 \\ (C) The Psychonomic Society, Inc. 2020
}

\begin{abstract}
The current study examined animacy and paired-associate learning through a survival-processing paradigm (Nairne et al. in Journal of Experimental Psychology: Learning, Memory, and Cognition, 33(2), 263-273, 2007; Schwartz \& Brothers, 2014). English-speaking monolingual participants were asked to learn a set of new word translations to improve their chances of survival or to improve their study abroad experience. Animate and inanimate words were included in this task, to further examine animacy effects in cued recall paradigms (Popp \& Serra in Journal of Experimental Psychology: Learning, Memory, and Cognition, 42(2), 186-201, 2016; VanArsdall et al. in Experimental Psychology, 60(3), 172-178, 2013). Across sentence-completion, matching, and picture-naming tasks, learning was facilitated by the survival context, relative to the study abroad context and an intentional learning condition. Scenario ratings indicated this survival advantage could also be a function of higher imageability ratings for the survival context than for the study abroad context. Replicating previous findings with cued recall, inanimate words were overall better remembered than animate words, across all three tasks, though survival processing facilitated language-learning for both animate and inanimate categories. This 'reverse animacy effect' replicated previous findings by Popp and Serra (Journal of Experimental Psychology: Learning, Memory, and Cognition, 42(2), 186-201, 2016), showing animate words can interfere with a participant's ability to create associations with their words, including those in a new language. These results are discussed with regards to the widely-reliable survival and animacy advantages, with a particular emphasis on the role of imageability in this paradigm.
\end{abstract}

Keywords Adaptive memory $\cdot$ Survival $\cdot$ Paired-associate learning $\cdot$ Animacy $\cdot$ Imageability

\section{Introduction}

Recent work investigating the functional aspects of human memory has noted an impressive and reliable finding: Memory is optimized when a word is processed for its survival value (Nairne, Thompson, \& Pandeirada, 2007). In this paradigm, researchers present participants with a set of words, asking them to rate each word's relevance to a given scenario. For example, participants in a survival condition read the following instructions:

Stephanie A. Kazanas

skazanas@tntech.edu

1 Department of Counseling and Psychology, Tennessee Technological University, Box 5031, Cookeville, TN 38505, USA

2 Department of Psychology, State University of New York, University at Albany, Social Sciences 399, 1400 Washington Avenue, Albany, NY 12222, USA
In this task, we would like you to imagine that you are stranded in the grasslands of a foreign land, without any basic survival materials. Over the next few months, you'll need to find steady supplies of food and water and protect yourself from predators. We are going to show you a list of words, and we would like you to rate how relevant each of these words would be for you in this survival situation. Some of the words may be relevant and others may not—it's up to you to decide (p. 264).

Participants in a similarly self-referent and schemaoriented moving condition read the following instructions:

In this task, we would like you to imagine that you are planning to move to a new home in a foreign land. Over the next few months, you'll need to locate and purchase a new home and transport your belongings. We are going to show you a list of words, and we would like you to rate how relevant each of these words would be for you in accomplishing this task. Some of the words may be relevant and others may not-it's up to you to decide (p. 264). 
Findings from recall and recognition tasks have supported a survival advantage: Numerous laboratories show words rated for their survival-relevance are more likely to be remembered than those rated for moving-relevance, pleasantness, or selfreference (e.g., Cho, Kazanas, \& Altarriba, 2018; Nairne, Pandeirada, \& Thompson, 2008; Nairne et al., 2007). These advantages have been reported for a variety of tasks, with the survival scenario pit against a range of control scenarios (Kazanas \& Altarriba, 2015; Nairne, Pandeirada, \& Fernandes, 2017). The survival advantage also replicates across both between- and within-subjects designs, though effect sizes can differ across these designs (Scofield, Buchanan, \& Kostic, 2018). A well-supported evolutionary account for the survival advantage suggests a general survival optimization system, wherein survival processing recruits a number of processes to encode those items, beyond those of other scenarios and instructions: elaboration and deep processing, simulations of future events, and so on (Nairne \& Pandeirada, 2016). This system highlights the prioritization of survival-relevant information, as was needed in our ancestral past. Support for this system has come from studies like those conducted by Kroneisen, Rummel, and Erdfelder (2014, 2016), who have found that dual-task settings and a high cognitive load can reduce and even eliminate the survival advantage, highlighting the degree of deep processing involved in these survival-relevance ratings.

Other researchers have argued against this evolutionary account for the survival advantage. Carefully-controlled experiments have shown the advantage can extend to scenarios describing supernatural predators (Kazanas \& Altarriba, 2017; Soderstrom \& McCabe, 2011) and unfamiliar locations (Kostic, McFarlan, \& Cleary, 2012). Though some question how an evolutionary account can accommodate these findings, Nairne and Pandeirada (2016) reason that these varied scenarios highlight the generalizability of self-preservation mechanisms. Yet, some cognitive mechanisms are still plausible. For one, survival processing appears to rely on both item-specific and relational processing — as evidenced by participants' recall—while other conditions encourage only itemspecific processing (Burns, Burns, \& Hwang, 2011; Burns, Hart, Griffith, \& Burns, 2013). In addition, richness-ofencoding explanations are well-supported: Participants engaging in survival processing generate significantly more ideas for each item they are given, with this added richness translating to better recall (e.g., Kroneisen \& Erdfelder, 2011; Röer, Bell, \& Buchner, 2013). Recent work by Fiacconi, Dekraker, and Köhler (2015) has also suggested an additional, arousal component, with participants' self-report and physiological data supporting this notion. These theoretical contributions, aimed at identifying ultimate (the 'why') and proximate (the 'how') mechanisms, are on-going.

A relatively new area of research that extends the original efforts of Nairne et al. (2007) investigates the relationship between animacy and memory (for a recent review, see
Altarriba \& Kazanas, 2019). Using the evolutionary account for the survival advantage, we might expect that the survival advantage would generalize to other fitness-related domains. For example, we know from the attention literature animate objects capture and maintain our attention more so than inanimate objects (Kazanas \& Altarriba, 2018), particularly when those objects represent a moving threat (Carretié et al. 2009). VanArsdall, Nairne, Pandeirada, and Blunt (2013) originally tested memory for nonwords that were presented with either a living property (e.g., dislikes tomatoes) or a nonliving property (e.g., requiring a key). For example, the nonword FRAV was presented, with the property 'has a round shape' presented below it. Participants decided whether each nonword was living or nonliving and they were instructed to remember the words for a later memory test. Recognition (Experiment 1) and recall (Experiment 2) memory were higher for nonwords presented with living properties. These findings were novel, in that, they were the first to support a mnemonic advantage for animacy, suggesting the human memory system may be guided by, or 'tuned' toward living properties: garnering additional support for the survival advantage.

In a related study, Nairne, VanArsdall, Pandeirada, Cogdill, and LeBreton (2013) reexamined results from an earlier word recall investigation. In their work, Rubin and Friendly (1986) had examined the lexical characteristics contributing to recall performance, reporting predictors including imagery, availability, and emotionality, but not animacy. Nairne et al. (2013) collected animacy ratings and found animacy may be the best predictor of recall. In fact, the positive correlation with recall was twice that of imagery. They extended these findings with an intentional learning task for animate and inanimate words (matched on nearly a dozen lexical variables). Recall was higher for the animate words, lending support to the earlier findings with nonwords presented by VanArsdall et al. (2013).

Additional support for this animacy advantage comes from a series of experiments conducted by Bonin, Gelin, and Bugaiska (2014). Their first two experiments included categorization and surprise free recall tasks. In these experiments, participants categorized French words (Experiment 1) or pictures (Experiment 2) as either animate or inanimate. In both experiments, participants categorized the animate stimuli faster than the inanimate stimuli. In addition, recall was higher for animate stimuli than for inanimate stimuli (with no differences in the number of intrusions). Experiment 3 used a recognition task, with the words from Experiment 1. Participants also indicated whether they 'remembered', 'knew', or 'guessed' when responding. Overall, animate words were recognized faster, with participants being more confident that they remembered the animate words from the categorization task (see also Li, Jia, Li, \& Li, 2016). Finally, in Experiment 4, the authors tested the possibility that this animacy effect may be due, in part, to animate words being more semantically 
rich. For example, semantically-rich concepts may have more features or contexts, evoke more sensations, or have more dense semantic neighborhoods (Pexman, Siakaluk, \& Yap, 2013). Although the words had been originally matched on imagery ratings, Bonin et al. (2014) collected a set of independent ratings for sensory experience: Participants rated each word according to its ability to evoke taste, touch, sight, sound, or smell, from 1 (no sensorial experience) to 7 (high sensorial experience). These ratings were equivalent for animate and inanimate words, reducing the likelihood that differences in memory for animate and inanimate words are a function of sensory experience. Related findings have suggested that these animacy advantages are not the result of animate words garnering higher processing fluency (Li et al., 2016), categorical structure (VanArsdall, Nairne, Pandeirada, \& Cogdill, 2017), or mental arousal (Meinhardt, Bell, Buchner, \& Röer, 2018; Popp \& Serra, 2018), nor do they rely on deeper processing (Bonin, Gelin, Laroche, Méot, \& Bugaiska, 2015; Leding, 2018), or are limited to particular encoding tasks (Gelin, Bugaiska, Méot, \& Bonin, 2017). For example, Gelin et al. (2017) had found robust animacy advantages in free recall across both survival and control scenarios. Importantly, animacy effects persist across other paradigms, too, including participants' enhanced memory for spatial and temporal information related to animate items when compared with inanimate items (Gelin, Bonin, Méot, \& Bugaiska, 2018). The attention afforded to animate items can also create more interference, as evidenced by larger Stroop effects with animate than inanimate words (Bugaiska et al., 2019). Together, these findings highlight the attention-grabbing nature of animate stimuli.

Other recent investigations from this literature have used paired-associate learning to test the survival and animacy advantages. In the first of these, Schwartz and Brothers (2014) conducted four experiments with participants studying either Swahili-English or Lithuanian-English word pairs. Utilizing both intentional learning and incidental learning paradigms, they consistently found better memory following pleasantness ratings for these pairs, relative to survivalrelevance ratings. Their findings provided one of the early limitations of the survival advantage (but see also Savine, Scullin, \& Roediger, 2011; Tse \& Altarriba, 2010) ${ }^{1}$. Popp and Serra (2016) had similar difficulties replicating the animacy advantage with paired-associate learning. Across their three experiments, they showed typical animacy advantages with free recall, but not with cued recall. They argued that these differences could not be a function of their stimulior they would not have replicated previous free recall

\footnotetext{
${ }^{1}$ Critically, Savine et al. (2011) and Tse and Altarriba (2010) did fail to replicate the survival advantage, though their tasks and stimuli greatly differed from the more familiar word recall procedure and this paired-associate learning procedure.
}

findings - but could perhaps be more indicative of some indirect effect between animacy and memory, perhaps aided by attention capture or mental arousal (but see Popp \& Serra, 2018). For example, an arousing animate word, such as a predator, would attract a participant's attention to that particular word, while also disrupting their ability to create and maintain a meaningful connection with its paired word. This effect would be magnified when the paired word is in an unfamiliar language and lacks its own retrieval cues (e.g., association strength, frequency). Similar predictions are made by Mather and Sutherland's (2011) arousal-biased competition $(\mathrm{ABC})$ theory: Arousal biases attention, directing mental resources from early perceptual processes to long-term memory consolidation, at the expense of low priority information (e.g., low survival value, goal-irrelevant).

Meanwhile, VanArsdall, Nairne, Pandeirada, and Cogdill (2015) have replicated their published animacy advantages using a cued recall paradigm (Nairne et al., 2013; VanArsdall et al., 2013). In their experiments, they selected animate and inanimate words from previous survival processing research (Nairne et al., 2013), as well as defined categories (four-legged animals and furniture), finding similar animacy advantages across these differing stimuli. As they discussed their findings, and animacy advantages more generally, they surmise that added richness, or even distinctiveness, could enhance the memorability of animate stimuli. Together, these findings with paired-associate learning and cued recall tasks are among the most inconsistent within the survival and animacy literatures. Popp and Serra (2016) argue that typical recall paradigms rely on individual item processing (as is the case with reported animacy advantages), while cued recall paradigms are at a disadvantage, because of the attentiongrabbing nature of the animate word in each word pair.

\section{The Current Study}

The following experiment expanded upon the survival and animacy literatures, using paired-associate learning, visual and auditory study materials, and a set of cued recall tasks. First, we modified the survival and moving scenarios to resemble more relevant language-learning settings for our participants (extending Schwartz \& Brothers, 2014). Emphasizing the importance of learning the word pairs, these scenarios engage intentional, deep processing, as did the intentional learning control condition. In addition, three cued recall tasks were created to examine the generalizability of the animacy effect (Nairne et al., 2013; VanArsdall et al., 2013, 2015) to other cued recall tasks: a sentence-completion task, picture-naming task, and matching task. Testing new variants of cued recall tasks is critical to understanding the conditions in which animacy can improve memory (i.e., a kind of 'task effect'). Moreover, conceptual replications of this kind remind us of the importance of science as self-correcting over time, as psychologists positively 
respond to calls for replication efforts (e.g., the Open Science Framework). Together, these new tasks explored and compared the extent of participants' learning across animate and inanimate word pairs, with survival, moving, and intentional learning conditions. Most importantly, they provide insight into the varied results surrounding animacy effects: a bit of a doubleedged sword that aids free recall and hinders cued recall.

Survival and animacy effects are well-supported by the relevant literatures, with a number of independent laboratories replicating these advantages. However, their ultimate and proximate mechanisms are still debated. Aiming to better understand these mechanisms, the current study also examined a series of mechanisms previously recommended by Nairne and Pandeirada (2010), namely, imageability and others (see also Otgaar et al., 2011; Sandry, Trafimow, Marks, \& Rice, 2013).

\section{Method}

\section{Participants}

One hundred and twenty University at Albany, State University of New York undergraduate students (53 males, 67 females) participated in this experiment, each a minimum of 18 years old $(M=19.76, S D=2.18)$. Each participant gave their informed consent and received course credit or extra credit compensation for the hour-long experiment. We used a brief demographic questionnaire to ensure each of our participants had no previous Spanish language instruction ${ }^{2}$.

\section{Research Design}

The current study employed a mixed design, with two independent variables. Scenario instructions were manipulated between-subjects, with participants randomly-assigned to learn word pairs according to either survival- or moving-processing, or for intentional learning purposes. Animacy was manipulated within-subjects, with all participants learning animate and inanimate word pairs. In this study, we adopted the following, binary distinction for animate and inanimate word pairs: Words representing animals (e.g., duck) were labeled 'animate' and words representing natural and human-made objects were labeled 'inanimate' (e.g., cherry, axe) (Popp \& Serra, 2016, 2018). Learning was measured with three tasks: sentence-completion, picture-naming, and matching.

\footnotetext{
$\overline{{ }^{2} \text { An additional }} 30$ students had participated in the experiment, but their language history indicated some previous language-learning experience with another Romance language (e.g., French); because there were so few of these participants divided across the three conditions, we omitted their data from these analyses.
}

\section{Materials}

A set of 24 concrete nouns (12 animate; 12 inanimate) were selected for this experiment, across a variety of common categories (from Van Overschelde, Rawson, \& Dunlosky, 2004). Animate categories included four-legged animals, insects, and birds. Inanimate categories included articles of clothing, fruits, and weapons (see Appendix 1 for the complete list of words). Animate and inanimate words were translated from English to Spanish and matched on number of letters, frequency, familiarity, number of syllables, imagery, and concreteness in both languages (see Table 1 for these means) (all $p \mathrm{~s}>.05$ ). In English, the English Lexicon Project (ELP; Balota et al., 2007) was utilized to match words on number of letters, frequency, and the number of syllables. Familiarity and imagery were matched using the MRC Psycholinguistic Database (Wilson, 1988). Finally, concreteness ratings were matched according to Brysbaert, Warriner, and Kuperman's (2014) concreteness database. In Spanish, frequency, familiarity, number of syllables, and imagery were matched using the BuscaPalabras (Davis \& Perea, 2005) database. The number of letters was matched according to Pérez, Alameda, and Cuetos's (2003) database, while concreteness ratings were matched using EsPal (Duchon, Perea, Sebastián-Gallés, Martí, \& Carreiras, 2013).

For the learning phase of the experiment, three sets of instructions were created (adapted from Nairne et al., 2007, 2008). Special consideration was taken to emphasize the relevance of the survival and moving conditions to learning the word pair translations. These instructions largely differed from those of Schwartz and Brothers (2014), who had emphasized the relevance-rating ${ }^{3}$ and pleasantness rating tasks to their participants. In our experiment, instructions for participants in the intentional learning condition read:

In this task, we are going to teach you a list of SpanishEnglish translations. We would like you to try to remember the new Spanish words for a future memory test.

Instructions for participants in the survival condition read:

In this task, we would like you to imagine that you are stranded in the grasslands of a foreign land, without any basic survival materials. Over the past few months,

\footnotetext{
${ }^{3}$ For example, Schwartz and Brothers' (2014, Experiment 1) survivalgrasslands scenario read: "Imagine as best as possible that you are stranded in a foreign grassland, such as the plains of Africa. You are the only one in this environment and you have no survival materials. Over the course of a few months, you will need to find steady supplies of food and water and protect yourself from predators. We are going to show you a list of word pairs on a PowerPoint slide show. Each slide will display a single word in Swahili on the left side, and its English translation on the right side, for 5 seconds. Your task is to rate how relevant each of these words would be for you in this survival situation (on a scale from 1 to $5 ; 1$ being barely relevant and 5 being highly relevant). Some of the words on these slides may be relevant and some may not - it is up to you to decide" (p. 162).
} 
Table 1. Word pair matching: animate and inanimate means and SDs

\begin{tabular}{lllll}
\hline & \multicolumn{2}{l}{ English words } & & \multicolumn{2}{l}{ Spanish words } \\
\cline { 2 - 3 } & Animate & Inanimate & Animate & Inanimate \\
\hline Number of letters & $4.87(1.69)$ & $5.67(1.92)$ & $5.27(1.10)$ & $10.56(14.25)$ \\
Frequency & $8.50(1.52)$ & $8.68(1.27)$ & $5.98(.37)$ & $14.99(14.81)$ \\
Familiarity & $3.35(2.87)$ & $4.64(2.54)$ & $2.53(.52)$ & $5.72(.91)$ \\
Number of syllables & $1.47(.64)$ & $1.40(.63)$ & $5.38(2.22)$ & $5.56(.51)$ \\
Imagery & $5.80(.52)$ & $6.05(.29)$ & $6.04(.35)$ & $5.62(.91)$ \\
Concreteness & $4.87(.09)$ & $4.89(.13)$ & & \\
\hline
\end{tabular}

you've had to find steady supplies of food and water and protect yourself from predators. Today, you encountered a stranger and you must work together to guarantee your chances of survival. This stranger speaks Spanish, but you do not, so you will have to learn some words in their language. We are going to teach you a list of SpanishEnglish translations. We would like you to try to remember the new Spanish words for a future memory test.

Instructions for participants in the moving condition read:

In this task, we would like you to imagine that you are planning to study abroad next semester in Spain. Over the next few months, you'll need to locate and rent a new apartment and transport your belongings overseas. Today, you learned that you will be getting a new roommate who only speaks Spanish. You must learn some words in their language to improve your living situation and study abroad experience. We are going to teach you a list of Spanish-English translations. We would like you to try to remember the new Spanish words for a future memory test.

Auditory recordings of the English-Spanish word pairs were created using the Audacity audio editor and recorder program. Word pairs were read aloud by a native Spanish speaker (e.g., duck-pato), one at a time, trimmed, and converted to WAV files within the program. Simple, black and white depictions of each word were selected from the Snodgrass and Vanderwart (1980) norms and the Clipart database (https://openclipart.org/) ${ }^{4}$, to present simultaneously with the written word pair presentation and audio recordings (the use of both words and images in acquiring new vocabulary is supported by the second language acquisition literature; Altarriba \& Knickerbocker, 2011). E-Prime 1.2 software was used to program the experiment, with word pair presentation randomly selected, and each word pair presented three times with its corresponding auditory recording and image.

\footnotetext{
${ }^{4}$ These images are available upon request from the corresponding author.
}

For the testing phase of the experiment, three tasks of varying difficulty were created to assess learning. A sentence-completion task was created to assess deeper, contextual understanding. Cloze sentences were created, such that each sentence would be completed by inserting one of the newly-learned words at the very end of the sentence (see Appendix 2 for these sentences). Each sentence was written in English and participants were instructed to write both the English and Spanish word that would complete the sentence; only complete English-Spanish word pairs were marked 'correct'. These sentences were piloted with 20 undergraduate students, and their mean completion rate was $70 \%$. The picture-naming task presented each picture one at a time and required participants to recall the Spanish word. Finally, a matching task provided an alphabetized list of the English and Spanish words and required participants to locate the word pairs they had learned. With the English list presented on one side and Spanish list presented on the other, participants indicated which corresponding Spanish word completed the English-Spanish word pair (e.g., to complete the duckword pair, they would indicate $R$, as pato appeared $18^{\text {th }}$ on the list).

\section{Procedure}

All participants were tested individually within the Cognition and Language Laboratory. Participants were randomly-assigned to one of the three conditions $(N=$ 40 for the survival, moving, and intentional learning conditions). Condition-specific instructions were displayed on a computer screen and reinforced by the experimenter, who remained with each participant and assisted throughout the experiment. The learning phase was identical across all conditions and word pairs, and participants were given five seconds to read and listen to each word pair, study the black-and-white image, and rehearse each word pair after it was presented. An additional five seconds were allotted in between each 
word pair to allow for participant rehearsal. The experimenter reinforced these instructions throughout the learning phase, particularly when participants hesitated to rehearse. A brief break was provided halfway through the learning phase of the experiment, with its length controlled by the participant.

Before the testing phase, participants were reminded about the importance of their learning performance. Participants in the survival condition were told:

Remember, your survival in this foreign land depends on your ability to communicate with your partner in this new language.

Participants in the moving condition were told:

Remember, your success in the study abroad program depends on your ability to communicate with your roommate in this new language.

During the testing phases, participants completed the three tasks in the same order: sentence-completion, picture-naming, and matching. This fixed order ensured that exposure to the learning material could not affect later task performance, particularly a shallow processing task (e.g., matching), limiting participants' need to engage in deep processing during the sentence-completion task. When participants asked about the importance of spelling accuracy, they were encouraged to try their best; small spelling errors were permitted (e.g., adding an ' $s$ ' to pluralize a word). After the testing phase, participants in the survival and moving conditions also answered the following questions pertaining to their scenario (from Nairne \& Pandeirada, 2010): (1) How interesting was the scenario? (2) How easy was it for you to create an "image" of the scenario in your mind? (3) How emotionally arousing was the scenario? (4) How familiar are you with the situation described? Two additional questions were included in the questionnaire (from Otgaar et al., 2011; Sandry et al., 2013): (5) How distinctive, or unusual, was the scenario? (6) How rich in detail would you rate the scenario?

\section{Results}

\section{Task Effects}

First, participants in all three conditions were matched on age, gender, and race, with all participants reporting English as their first language (all $p \mathrm{~s}>.05$ ). Next, a series of 3 (Condition: intentional, moving, or survival) $\times 2$ (Animacy: animate and inanimate) mixed analyses of variance
(ANOVAs) were conducted for each task, with animacy examined as a repeated-measures variable ${ }^{5}$. Planned comparisons were conducted to examine differences across conditions (these findings are depicted in Fig. 1). Findings from the three tasks show a very similar pattern of results, with participants in the survival condition remembering significantly more animate and inanimate word pairs than those in the moving and intentional conditions. Significant, task-specific findings follow.

With the sentence-completion task, the main effect of condition was significant, $F(2,117)=10.856, p<.001, \eta_{\mathrm{p}}{ }^{2}=.157$, with participants in the survival condition $(M=.45)$ performing significantly better than those in the moving ( $M$ $=.32$ ) and intentional $(M=.29)$ conditions ( $p s<.01$; the latter two did not differ). The main effect of animacy was also significant, $F(1,117)=6.129, p<.05, \eta_{\mathrm{p}}^{2}=.050$, with better performance on inanimate sentences $(M=.37)$ than on animate sentences $(M=.33)$. The interaction between condition and animacy was not significant $(p>.05)$, though participants in the survival condition performed similarly well on animate $(M=.45)$ and inanimate sentences $(M=.44)$.

With the picture-naming task, the main effect of condition was significant, $F(2,117)=9.922, p<.001, \eta_{\mathrm{p}}{ }^{2}=.145$, with participants in the survival condition $(M=.48)$ performing significantly better than those in the moving $(M=.34)$ and intentional $(M=.34)$ conditions $(p s<.01$; the latter two did not differ). The main effect of animacy was also significant, $F(1,117)=19.015, p<.001, \eta_{\mathrm{p}}^{2}=.140$, with better performance on inanimate pictures $(M=.42)$ than on animate pictures $(M=.35)$. The interaction between condition and animacy was not significant $(p>.05)$, though, again, animate and inanimate naming were highest in the survival condition ( $M=.44$ and $M=.52$, respectively).

With the matching task, the main effect of condition was significant, $F(2,117)=5.326, p<.01, \eta_{\mathrm{p}}^{2}=.083$, with participants in the survival condition $(M=.77)$ performing significantly better than those in the intentional $(M=.63)$ condition $(p<.01$; the other condition comparisons were non-significant). The main effect of animacy was also significant, $F(1,117)=58.031, p<.001, \eta_{\mathrm{p}}{ }^{2}=.332$, with better performance on inanimate word pairs $(M=.75)$ than on animate word pairs $(M=.62)$. The interaction between condition and animacy was not significant $(p>.05)$, though, similar to the naming data, animate and inanimate word pair matching was highest in the survival condition $(M=.72$ and $M=.81$, respectively).

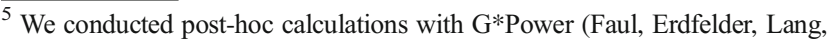
\& Buchner, 2007), estimating a small effect size, as reported by Scofield et al.'s (2018) meta-analysis. We found the interactions and main effect analyses to be well-powered (each $>.86$ ). The authors wish to thank an anonymous reviewer for recommending these additional analyses.
} 


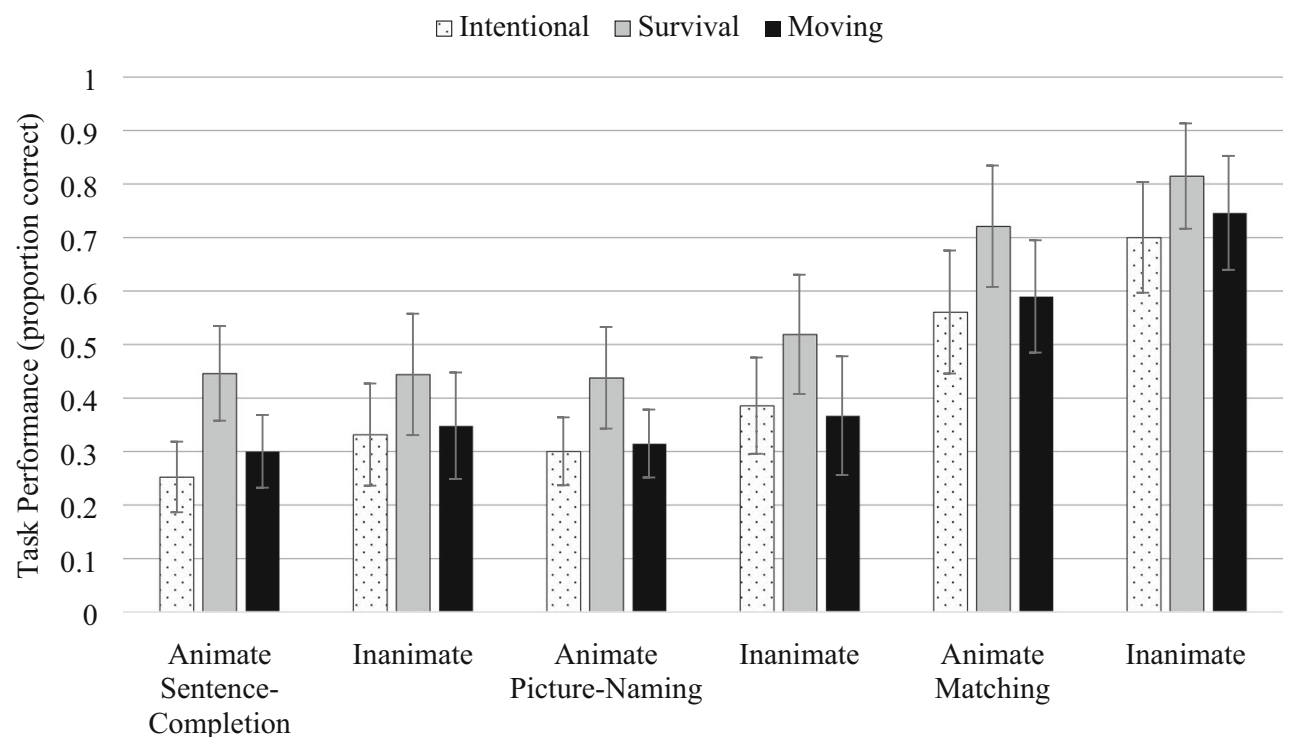

Fig. 1. A comparison of condition and animacy findings, with \pm 1 standard deviations (SDs) shown.

\section{Scenario Ratings}

Scenario ratings for the survival and moving conditions were also examined for their effect on task performance (all means and $S D$ s presented in Table 2). First, a series of independentsamples $t$-tests were conducted to detect any differences in each of the scenario ratings between the survival and moving conditions. Two of these tests were significant, after applying the Bonferroni correction. The comparison between imageability scores was significant, $t(78)=3.09, p<.01$, with the survival condition $(M=4.08, S D=.86)$ rated as more imageable than the moving condition $(M=3.38, S D=1.15)$. The comparison between distinctiveness scores was also significant, $t(78)=2.09, p<.05$, with the survival condition $(M=$ $3.23, S D=.83)$, rated as more distinctive than the moving condition $(M=2.75, S D=1.17)$. The ratings for interest, arousal, familiarity, and richness did not differ across the survival and moving conditions.

Next, correlation and multiple regression analyses were used to develop a model predicting participants' task performance from their condition and scenario ratings. These analyses

Table 2. Scenario rating means and SDs

\begin{tabular}{lll}
\hline Rating dimension & Survival & Moving \\
\hline Interest & $3.56(.71)$ & $3.68(1.00)$ \\
Imageability ** & $4.08(.86)$ & $3.38(1.15)$ \\
Arousal & $2.35(.89)$ & $2.53(1.01)$ \\
Familiarity & $1.98(.95)$ & $2.43(1.17)$ \\
Distinctiveness * & $3.23(.83)$ & $2.75(1.17)$ \\
Richness & $3.43(.81)$ & $3.38(0.84)$ \\
\hline
\end{tabular}

$* p<.05$

$* * p<.01$ provide both the particular magnitude and direction of these relationships. Findings from the three sets of analyses support the role of both condition and imageability in predicting task performance. Significant correlations and regression models follow. With the sentence-completion task, condition (coded as $0=$ moving, $1=$ survival) was positively correlated with task performance, $r(78)=.354, p=.001$, confirming that participants in the survival condition tended to have higher sentencecompletion scores. Imageability scores were also positively correlated with task performance, $r(78)=.337, p=.001$, indicating that higher imageability scores were also related to higher sentence-completion scores. The multiple regression model with condition and imageability predicted $18 \%$ of the variance in sentence-completion performance, $F(1,78)=11.18, p=.001$. Of the two significant predictors, participants' condition $(\beta=$ .273 ) accounted for a greater amount of unique variance in sentence-completion performance than did imageability scores ( $\beta=.247)$. Interest, arousal, familiarity, distinctiveness, and richness were not incorporated into the model.

Similar findings were observed with the picture-naming task, as condition was positively correlated with task performance, $r(78)=.387, p<.001$, again confirming that participants in the survival condition tended to have higher picturenaming scores. Imageability scores were also positively correlated with task performance, $r(78)=.423, p<.001$, indicating that higher imageability scores were related to higher picturenaming performance. The multiple regression model with these variables predicted $25 \%$ of the variance in picture-naming performance, $F(1,78)=17.02, p<.001$. Of the two significant predictors, imageability scores $(\beta=.332)$ accounted for a greater amount of unique variance in picture-naming performance than did participants' condition ( $\beta=.277)$, as we might expect, given the nature of the task. The other rating dimensions were not incorporated into the model. 
Finally, with the matching task, condition was positively correlated with task performance, $r(78)=.257, p<.05$, also confirming that participants in the survival condition tended to have higher matching scores. Imageability scores were also positively correlated with task performance, $r(78)=.280, p<$ .01 , indicating that higher imageability scores were related to higher matching task performance. However, the regression model retained only participants' imageability scores, predicting $7 \%$ of the variance in matching task performance, $F(1,78)=6.63, p<.05$. Participant condition and the other rating dimensions were not incorporated into the model. Together, findings from these regression analyses largely support the role of imageability, as it enhanced performance on all three tasks used in the experiment.

\section{Discussion}

The current study aimed to further investigate the generalizability of both the survival and animacy advantages reported in the memory literature (e.g., Nairne et al., 2007, 2008; VanArsdall et al., 2013, 2015, 2017). These advantages illustrate the value and prioritization of fitness-relevant information in human cognition. To test these advantages within a paired-associate learning paradigm, three sets of instructions were created and manipulated between-subjects: One represented an intentional learning condition and two described scenarios that necessitated foreign language learning (for survival and moving purposes). The to-be-learned material included both animate and inanimate word pairs, with a novel visual and audio presentation. Participants' learning was tested via three tasks: sentence-completion, picture-naming, and matching. These tasks represent new measures for both the survival- and animacy-processing literatures, developed to better understand the generalizability of these advantages beyond standard recall procedures. These novel approaches can reveal both generalizations and limitations - as was the case with the current study.

Consistent with the survival advantage literature (Kazanas \& Altarriba, 2015), we detected a robust survival advantage with all three tasks, across both animate and inanimate word pairs. These results differ from those reported by Schwartz and Brothers (2014) in their investigation of the survival advantage within paired-associate learning. We would argue that our modified scenarios - and omitting the relevance-rating task-likely heightened participants' focus on learning, as they engaged in the deep processing element of the task. Perhaps that focus, or the emphasis on intentional learning implied in the scenario, in turn heightened the survival advantage (see Nairne, VanArsdall, Pandeirada, \& Blunt, 2012 for a similar argument for location memory advantages following survival processing).
Despite this reliable survival advantage, these data do not support a typical animacy advantage: one in which animacy enhances retention. Across each of the tasks, a consistent advantage for the inanimate word pairs was observed, depicted in each of the three main effects for animacy word type. These findings do not replicate previous animacy advantages observed with recall and recognition measures (e.g., Aslan \& John, 2016; Bonin et al., 2014; Gelin et al., 2017; Li et al., 2016; Popp \& Serra, 2018; VanArsdall et al., 2017). However, they do replicate other recent findings suggesting a limitation to the animacy advantage: one that lies in pairedassociate learning (Popp \& Serra, 2016). Consistent with Popp and Serra's findings, animacy appeared to impair cued recall performance, and this effect was observed with sentence, picture, and word cues. They have argued that animacy has a negative effect on most paired-associate tasks because a participant's attention is quickly drawn to only one of the words (the animate word); attention capture limits their ability to associate the animate word with another word. Inanimate words are less likely to create this interference, as is typical of low priority information and less-arousing stimuli (Mather \& Sutherland, 2011). Careful matching across languages and animacy word type reduces the likelihood that any particular lexical variable can account for this reverse animacy effect. In addition, the similarity of findings observed across the three tasks and those by Popp and Serra (2016) fall in line with explanations proposed by both Popp and Serra (2016) and VanArsdall et al. (2015): The added richness and attention-grabbing nature of animate stimuli promote deeper processing benefitting free recall, but hinder the associative processing needed in cued recall.

The scenario rating data also replicate previous rating differences across survival and control conditions. For example, participants in Nairne and Pandeirada's (2010) early research described an ancestral version of the survival scenario as more unusual than a modern version of the survival scenario. These rating differences were corroborated by better memory for words rated for their ancestral survival-relevance. Otgaar et al. (2011) reported similar findings, with their survival advantage supported by familiarity and richness ratings. The survival scenario was rated high in richness and low in familiarity, again showing an effect of distinctiveness. More recently, our laboratory has shown effects of scenario interest and imageability, with participants rating the survival scenario as both more interesting and more imageable than the other scenarios (Kazanas \& Altarriba, 2017; Kazanas, Van Valkenburg, \& Altarriba, 2015). Imageability may then play a role in observed differences in distinctiveness ratings, and vice versa (i.e., the survival scenario may be perceived as more imageable because of its distinctiveness). Kroneisen and Erdfelder (2011) have further argued that these components are just two of the many mechanisms 
defining a richness-of-encoding hypothesis for the survival advantage: that the survival scenario promotes deeper processing during encoding and provides numerous retrieval cues, relative to other scenarios and processing tasks (see also Kroneisen, Erdfelder, \& Buchner, 2013).

In the current study, ANOVA and correlational analyses with the scenario data indicate a strong relationship between the survival scenarios' imageability and its effect on paired-associate learning. Across all three tasks, imageability predicted task performance; these findings were also supported by overall differences between the survival and moving scenarios, with the survival scenario rated as more imageable and more distinctive than the moving scenario. Together, these differences in scenario ratings support both the earlier research claiming a difference in distinctiveness among the scenarios (Nairne \& Pandeirada, 2010; Otgaar et al., 2011) and the more recent research highlighting the role of imageability in the survival advantage (Kazanas \& Altarriba, 2017; Kazanas et al., 2015). In the end, it may be the case that the survival scenario primes, or prompts a general survival optimization system, such as the one described by Nairne and Pandeirada (2016): a system that enhances human cognition because a salient situation, like one threatening a person's survival, is also more likely to be highly imageable, distinctive, and so on. These scenario data largely support that optimization system and its proximate mechanisms (for a review of these and other mechanisms, see Nairne \& Pandeirada, 2016).

Recent findings from other laboratories suggest imagery and attention processes may play a role in animacy advantages. In one, early example, Bonin et al. (2015) claimed that imagery processes do contribute to animacy effects. Their Study 4 asked participants to engage in interactive imagery as they encoded animate and inanimate words (e.g., "If you see the word FORK, you could imagine yourself in your kitchen, using the fork to eat," p. 379). They found that memory for the inanimate words was boosted by the interactive imagery instructions, whereas animate words did not benefit from those same instructions. These findings suggest an important relationship, perhaps some mediation, between imagery processes and the animacy effect (though some more recent findings argue against this hypothesis; Gelin, Bugaiska, Méot, Vinter, \& Bonin, 2019).

We encourage future studies in this area of research to adopt similar goals: testing these memory and processing advantages with other stimuli, tasks, and procedures. When researchers opt for numerous tasks, they should be careful to limit carryover effects with their data. With some tasks, carryover effects can be eliminated with counterbalancing procedures ${ }^{6}$. Studying the applied nature and generalizability of adaptive memory-including the recent branches of animacy, contamination, and face memory-will increase our understanding of and confidence in Nairne and Pandeirada's (2016) general survival optimization system. For example, recent investigations have shown that survival processing can, indeed, promote better memory for potential mate partners (Pandeirada, Fernandes, Vasconcelos, \& Nairne, 2017) and contaminated objects (Bonin, Thiebaut, Witt, \& Méot, 2019; Fernandes, Pandeirada, Soares, \& Nairne, 2017), as well as enhance associative processing (Dewhurst, Anderson, Grace, \& van Esch, 2016) and activate typical neurobiological fear responses (Fiacconi et al., 2015). These applications represent an important intersection between the evolutionary and cognitive psychology literatures, as we explore a functionalist explanation for human cognition.

\section{Conclusions}

The current study investigated the survival and animacy advantages with paired-associate learning, with findings supporting a survival advantage with three cued recall tasks, but no animacy advantage observed with any of the three cued recall tasks. These results support the widely-reliable survival advantage (Kazanas \& Altarriba, 2015), with a set of scenarios, stimuli, and tasks adapted for language-learning. Failing to replicate the animacy advantage with cued recall, these results support an attention-grabbing mechanism specific to animate words that hinders associative processing, but not free recall performance (Popp \& Serra, 2016). We argue that some (perhaps significant) portion of the animacy effect relies on animate stimuli grabbing and maintaining attention, perhaps at the expense of other inanimate words or cues; this explanation can account for both free and cued-recall data. These findings, and their replication and extension of previous works, emphasize the importance of applying new and adapted materials and

\footnotetext{
${ }^{6}$ We would like to thank an anonymous reviewer for this recommendation. The current study necessitated a fixed order to ensure limited carryover effects for each of the three tasks. For example, deeper processing for sentencecompletion would not be necessary if the word pair had been activated during picture-naming or matching. Despite this limitation, post-hoc analyses for matching task performance, conducted with 'new items' (those produced during the matching test, but not prior) largely mimicked overall findings, with greater performance on inanimate word pairs $(M=.39)$ than on animate word pairs $(M=.36)$. Though this difference was not significant, it is consistent with the 'reverse animacy effect' we replicated across the three tasks. Future researchers might also consider adopting a fully between-subjects design for their study.
} 
tasks to these paradigms. These applications, such as the adaptive value of language-learning for collaboration and safety in survival settings, highlight the generalizability of the survival advantage.

Author Note Portions of these data were presented at the 58th annual meeting of The Psychonomic Society, Vancouver, BC. We would like to thank David Fikhman and Shirell Favor for their assistance with data coding and entry and Halszka Bąk and Allison Wilck for coordinating these efforts.

Open Practices Statement Though this experiment was not preregistered, data and analyses are available upon request from the corresponding author.

\section{Appendix 1}

\begin{tabular}{cccc}
\hline Animate words & \multicolumn{3}{c}{ Inanimate words } \\
\hline $\begin{array}{c}\text { English } \\
\text { ant }\end{array}$ & Spanish & English & Spanish \\
bear & hormiga & apple & manzana \\
chicken & oso & axe & hacha \\
cow & pollo & cherry & cereza \\
dog & vaca & coat & abrigo \\
duck & perro & dress & vestido \\
flea & pato & glove & guante \\
fly & pulga & grape & uva \\
seagull & mosca & hammer & martillo \\
sheep & gaviota & knife & cuchillo \\
spider & oveja & skirt & falda \\
worm & araña & strawberry & fresa \\
\hline
\end{tabular}

\section{Appendix 2}

1. If you're going to help me chop down the tree, fetch me the

2. Let's go to the dairy farm and learn how to milk a

3. When I sit by the pond, my favorite activity is feeding the

4. Before you go play in the snow, put on your hat and

5. We're scared of birds at the beach particularly the ones that take your food like

6. When you visit your aunt in Alaska this winter, remember to pack a heavy

7. Before you head to school this morning, don't forget to walk the
8. Jenna should start shopping now if she wants to find the perfect prom

9. "Remember, only you can prevent forest fires", says Smokey the

10. At the end of the year, all of the students brought their teacher an

11. At Mary's private school, girls were required to wear pants or a knee-length

12. We always know that Aunt Betsy's favorite shortcake is c overed in red, juic y

13. Let's get creepy Halloween decorations for the party: cauldrons, webs, and a big, hairy

14. In the King Arthur legend, Excalibur was the name of Arthur's

15. Let's finish making this sundae by topping it with a maraschino

16. While some wool comes from goats and camels, most comes from

17. Before you cast your line, grab the fishing hook and put on a

18. She had a picnic lunch spread at the park but was attacked by hungry

19. Let's go to the pet store and get Barkley some medicine to prevent ticks and

20. You can help me cut these vegetables by passing the sharp

21. A good handyman's toolbox should always have some nails and a

22. Betty is so kind to animals that she wouldn't even hurt a

23. Check the coop and see if there are any fresh eggs from the

24. To finish the fruit salad, I need you to buy a big bunch of seedless

\section{References}

Altarriba, J., \& Kazanas, S. A. (2019). Animacy and mortality salience: New directions for the adaptive memory literature. In T. K. Shackelford \& V. Zeigler-Hill (Eds.), Evolutionary perspectives on death (pp. 63-76). Cham, Switzerland: Springer.

Altarriba, J., \& Knickerbocker, H. (2011). Acquiring second language vocabulary through the use of images and words. In P. Trofimovich \& R. McDonough (Eds.), Applying priming methods to L2 learning, teaching, and research (pp. 21-47). Amsterdam: John Benjamins.

Aslan, A., \& John, T. (2016). The development of adaptive memory: Young children show enhanced retention of animacy-related information. Journal of Experimental Child Psychology, 152, 343-350. 
Balota, D. A., Yap, M. J., Cortese, M. J., Hutchison, K. A., Kessler, B., Loftis, B., ... \& Treiman, R. (2007). The English Lexicon Project. Behavior Research Methods, 39, 445- 459.

Bonin, P., Gelin, M., \& Bugaiska, A. (2014). Animates are better remembered than inanimates: Further evidence from word and picture stimuli. Memory and Cognition, 42, 370-382.

Bonin, P., Gelin, M., Laroche, B., Méot, A., \& Bugaiska, A. (2015). The "how" of animacy effects in episodic memory. Experimental Psychology, 62(6), 371-384.

Bonin, P., Thiebaut, G., Witt, A., \& Méot, A. (2019). Contamination is "good" for your memory! Further evidence for the adaptive view of memory. Evolutionary Psychological Science, 5(3), 300-316.

Bugaiska, A., Grégoire, L., Camblats, A.-M., Gelin, M., Méot, A., \& Bonin, P. (2019). Animacy and attentional processes: Evidence from the Stroop task. Quarterly Journal of Experimental Psychology, 72(4), 882-889.

Burns, D. J., Burns, S. A., \& Hwang, A. J. (2011). Adaptive memory: Determining the proximate mechanisms responsible for the memorial advantages of survival processing. Journal of Experimental Psychology: Learning, Memory, and Cognition, 37, 206-218.

Burns, D. J., Hart, J., Griffith, S. E., \& Burns, A. D. (2013). Adaptive memory: The survival scenario enhances item-specific processing relative to a moving scenario. Memory, 21, 695-706.

Brysbaert, M., Warriner, A. B., \& Kuperman, V. (2014). Concreteness ratings for 40 thousand generally known English word lemmas. Behavior Research Methods, 46(3), 904-911.

Carretié, L., Hinojosa, J. A., López-Martín, S., Albert, J., Tapia, M., \& Pozo, M. A. (2009). Danger is worse when it moves: Neural and behavioral indices of enhanced attentional capture by dynamic threatening stimuli. Neuropsychologia, 47, 364-369.

Cho, K. W., Kazanas, S. A., \& Altarriba, J. (2018). Survival processing in recognition memory: Separating recollection from familiarity. American Journal of Psychology, 131(1), 19-32.

Davis, C. J., \& Perea, M. (2005). BuscaPalabras: A program for deriving orthographic and phonological neighborhood statistics and other psycholinguistic indices in Spanish. Behavior Research Methods, 37(4), 665-671.

Dewhurst, S. A., Anderson, R. J., Grace, L., \& van Esch, L. (2016). Adaptive false memory: Imagining future scenarios increases false memories in the DRM paradigm. Memory \& Cognition, 44(7), 1076-1084.

Duchon, A., Perea, M., Sebastián-Gallés, N., Martí, A., \& Carreiras, M. (2013). EsPal: One-stop shopping for Spanish word properties. Behavior Research Methods, 45(4), 1246-1258.

Faul, F., Erdfelder, E., Lang, A.-G., \& Buchner, A. (2007). G*Power 3: A flexible statistical power analysis program for the social, behavioral, and biomedical sciences. Behavior Research Methods, 39, 175-191.

Fernandes, N. L., Pandeirada, J. N. S., Soares, S. C., \& Nairne, J. S. (2017). Adaptive memory: The mnemonic value of contamination. Evolution and Human Behavior, 38(4), 451-460.

Fiacconi, C. M., Dekraker, J., Köhler, S. (2015). Psychophysiological evidence for the role of emotion in adaptive memory. Journal of Experimental Psychology: General, 144(5), 925-933.

Gelin, M., Bonin, P., Méot, A., \& Bugaiska, A. (2018). Do animacy effects persist in memory for context? Quarterly Journal of Experimental Psychology, 71(4), 965-974.

Gelin, M., Bugaiska, A., Méot, A., \& Bonin, P. (2017). Are animacy effects in episodic memory independent of encoding instructions? Memory, 25(1), 2-18.

Gelin, M., Bugaiska, A., Méot, A., Vinter, A., \& Bonin, P. (2019). Animacy effects in episodic memory: Do imagery processes really play a role? Memory, 27(2), 209-223.

Kazanas, S. A., \& Altarriba, J. (2018). Predators as attention-grabbing. In T. Shackelford \& V. Weekes-Schackelford (Eds.)., Encyclopedia of evolutionary psychological science. Springer International Publishing.
Kazanas, S. A., \& Altarriba, J. (2017). Did our ancestors fear the unknown? The role of predation in the survival advantage. Evolutionary Behavioral Sciences, 11(1), 83-91.

Kazanas, S. A., \& Altarriba, J. (2015). The survival advantage: Underlying mechanisms and extant limitations. Evolutionary Psychology, 13(2), 360-396.

Kazanas, S. A., Van Valkenburg, K. M., \& Altarriba, J. (2015). Survival processing and the Stroop task: Does the survival advantage depend on deeper processing during encoding? Evolutionary Psychology, 13(4), 1-8.

Kostic, B., McFarlan, C. C., \& Cleary, A. M. (2012). Extensions of the survival advantage in memory: Examining the role of ancestral context and implied social isolation. Journal of Experimental Psychology: Learning, Memory, and Cognition, 38, 1091-1098.

Kroneisen, M., \& Erdfelder, E. (2011). On the plasticity of the survivalprocessing effect. Journal of Experimental Psychology: Learning, Memory, and Cognition, 37(6), 1552- 1563.

Kroneisen, M., Erdfelder, E., \& Buchner, A. (2013). The proximate memory mechanism underlying the survival-processing effect: Richness of encoding or interactive imagery? Memory, 21(4), 494-502.

Kroneisen, M., Rummel, J., \& Erdfelder, E. (2014). Working memory load eliminates the survival processing effect. Memory, 22(1), 92-102.

Kroneisen, M., Rummel, J., \& Erdfelder, E. (2016). What kind of processing is survival processing? Memory \& Cognition, 44(8), 1228-1243.

Leding, J. K. (2018). The animacy advantage in memory: Manipulations of levels of processing and survival processing. American Journal of Psychology, 131(3), 273-281.

Li, P., Jia, X., Li, X., \& Li, W. (2016). The effect of animacy on metamemory. Memory and Cognition, 44, 696-705.

Mather, M., \& Sutherland, M. R. (2011). Arousal-biased competition in perception and memory. Perspectives on Psychological Science, $6(2), 114-133$

Meinhardt, M. J., Bell, R., Buchner, A., Röer, J. P. (2018). Adaptive memory: Is the animacy effect on memory due to emotional arousal? Psychonomic Bulletin \& Review, 25(4), 1399-1404.

Nairne, J. S., \& Pandeirada, J. N. (2010). Adaptive memory: Ancestral priorities and the mnemonic value of survival processing. Cognitive Psychology, 61(1), 1-22.

Nairne, J. S., \& Pandeirada, J. N. S. (2016). Adaptive memory: The evolutionary significance of survival processing. Perspectives on Psychological Science, 11(4), 496-511.

Nairne, J. S., Pandeirada, J. N. S., \& Fernandes, N. L. (2017). Adaptive memory. In Byrne, J. H. (Ed.), Learning and memory: A comprehensive reference (pp. 279-293). Oxford, England: Academic Press.

Nairne, J. S., Pandeirada, J. N. S., \& Thompson, S. R. (2008). Adaptive memory: The comparative value of survival processing. Psychological Science, 19(2), 176-180.

Nairne, J. S., Thompson, S. R., \& Pandeirada, J. N. S. (2007). Adaptive memory: Survival processing enhances retention. Journal of Experimental Psychology: Learning, Memory, and Cognition, 33(2), 263-273.

Nairne, J. S., VanArsdall, J. E., Pandeirada, J. N. S., \& Blunt, J. R. (2012). Adaptive memory: Enhanced location memory after survival processing. Journal of Experimental Psychology: Learning, Memory, and Cognition, 38(2), 495-501.

Nairne, J. S., VanArsdall, J. E., Pandeirada, J. N. S., Cogdill, M., \& LeBreton, J. M. (2013). Adaptive memory: The mnemonic value of animacy. Psychological Science, 24(10), 2099-2105.

Otgaar, H., Smeets, T., Merckelbach, H., Jelicic, M., Verschuere, B., Galliot, A.-M., \& van Riel, L. (2011). Adaptive memory: Stereotype activation is not enough. Memory \& Cognition, 39, 1033-1041.

Pandeirada, J. N. S., Fernandes, N. L., Vasconcelos, M., \& Nairne, J. S. (2017). Adaptive memory: Remembering potential mates. Evolutionary Psychology, 15(4), 1-11.

Pérez, M. Á., Alameda, J. R., \& Cuetos, F. (2003). Frecuencia, longitud y vecindad ortográfica de las palabras de 3 a 16 letras del Diccionario 
de la Lengua Española (RAE, 1992). Revista Electrónica de Metodología Aplicada, 8(2), 1-10.

Pexman, P. M., Siakaluk, P. D., \& Yap, M. J. (2013). Meaning in mind: Semantic richness effects in language processing. Frontiers in Human Neuroscience, 7, 5-7.

Popp, E. Y., \& Serra, M. J. (2016). Adaptive memory: Animacy enhances free recall but impairs cued recall. Journal of Experimental Psychology: Learning, Memory, and Cognition, 42(2), 186-201.

Popp, E. Y., \& Serra, M. J. (2018). The animacy advantage for free-recall performance is not attributable to greater mental arousal. Memory, 26(1), 89-95.

Röer, J. P., Bell, R., \& Buchner, A. (2013). Is the survival-processing memory advantage due to richness of encoding? Journal of Experimental Psychology: Learning, Memory, and Cognition, 39, 1294-1302.

Rubin, D. C., \& Friendly, M. (1986). Predicting which words get recalled: Measures of free recall, availability, goodness, emotionality, and pronounceability for 925 nouns. Memory \& Cognition, 14(1), 79-94.

Sandry, J., Trafimow, D., Marks, M. J., \& Rice, S. (2013). Adaptive memory: Evaluating alternative forms of fitness-relevant processing in the survival processing paradigm. PloS one, 8(4), e60868.

Savine, A. C., Scullin, M. K., \& Roediger H. L., III. (2011). Survival processing of faces. Memory \& Cognition, 39(8), 1359-1373.

Schwartz, B. L., \& Brothers, B. R. (2014). Survival processing does not improve paired- associate learning. In B. L. Schwartz, M. L. Howe, M. P. Toglia, and H. Otgaar (Eds.), What is adaptive about adaptive memory? (pp. 159-181). New York: Oxford University Press.

Scofield, J. E., Buchanan, E. M., \& Kostic, B. (2018). A meta-analysis of the survival-processing advantage in memory. Psychonomic Bulletin \& Review, 25, 997-1012.
Snodgrass, J. G., \& Vanderwart, M. (1980). A standardized set of 280 pictures: Norms for name agreement, image agreement, familiarity, and visual complexity. Journal of Experimental Psychology: Human Learning and Memory, 6(2), 174-215.

Soderstorm, N. C., \& McCabe, D. P. (2011). Are survival processing memory advantages based on ancestral priorities? Psychonomic Bulletin \& Review, 18, 564-569.

Tse, C.-S., \& Altarriba, J. (2010). Does survival processing enhance implicit memory? Memory \& Cognition, 38(8), 1110-1121.

Van Overschelde, J. P., Rawson, K. A., \& Dunlosky, J. (2004). Category norms: An updated and expanded version of the norms. Journal of Memory and Language, 50(3), 289-335.

VanArsdall, J. E., Nairne, J. S., Pandeirada, J. N. S., \& Blunt, J. R. (2013). Adaptive memory: Animacy processing produces mnemonic advantages. Experimental Psychology, 60(3), 172-178.

VanArsdall, J. E., Nairne, J. S., Pandeirada, J. N. S., \& Cogdill, M. (2015). Adaptive memory: Animacy effects persist in pairedassociate learning. Memory, 23(5), 657-663.

VanArsdall, J. E., Nairne, J. S., Pandeirada, J. N. S., \& Cogdill, M. (2017). A categorical recall strategy does not explain animacy effects in episodic memory. The Quarterly Journal of Experimental Psychology, 70(4), 761-771.

Wilson, M. (1988). MRC psycholinguistic database: Machine-usable dictionary. Behavior Research Methods, 20(1), 6-10.

Publisher's Note Springer Nature remains neutral with regard to jurisdictional claims in published maps and institutional affiliations. 